\title{
Biomarker assay validation for clinical trials: a questionnaire survey to pharmaceutical companies in Japan
}

\author{
Yoshiaki Ohtsu ${ }^{\ddagger 1}$, Takehisa Matsumaru ${ }^{\ddagger 2}$, Masataka Katashima ${ }^{\ddagger}{ }^{3}$, Masaaki Kakehi ${ }^{4}$ \\ Hiroyuki Kakuo ${ }^{5}$, Takayoshi Suzuki ${ }^{6}$ Masanari Mabuchi ${ }^{7}$, Ryosuke Nakamura ${ }^{6}$, Takahiro \\ Nakamura ${ }^{8}$, Noriko Katori ${ }^{6}$, Seiji Tanaka ${ }^{9}$ \& Yoshiro Saito*,6 \\ ${ }^{1}$ Astellas Pharma Inc., Tsukuba, Ibaraki, Japan \\ ${ }^{2}$ Otsuka Pharmaceutical Co., Ltd, Osaka, Japan \\ ${ }^{3}$ Astellas Pharma Inc., Tokyo, Japan \\ ${ }^{4}$ Takeda Pharmaceutical Company Limited, Fujisawa, Kanagawa, Japan \\ ${ }^{5}$ Taiho Pharmaceutical Co., Ltd, Tsukuba, Ibaraki, Japan \\ ${ }^{6}$ National Institute of Health Sciences, Kawasaki, Kanagawa, Japan \\ ${ }^{7}$ Mitsubishi Tanabe Pharma Corporation, Yokohama, Kanagawa, Japan \\ ${ }^{8}$ Shin Nippon Biomedical Laboratories, Ltd, Kainan, Wakayama, Japan \\ ${ }^{9}$ Aska Pharmaceutical Co., Ltd, Kawasaki, Kanagawa, Japan \\ *Author for correspondence: yoshiro@nihs.go.jp \\ ${ }^{\ddagger}$ Authors contributed equally
}
"The present survey in Japan revealed that biomarker assays during clinical trials have become common in drug development and approximately $30 \%$ of the assays are for regulatory decision-making"

First draft submitted: 28 September 2018; Accepted for publication: 2 November 2018; Published online: 26 November 2018

Keywords: assay validation $\bullet$ biomarker $\bullet$ clinical trials $\bullet$ Japan $\bullet$ standard operating procedure

The pharmaceutical industry has been working on improving drug development since decades. Currently, lack of efficacy and safety concerns are the major causes of attrition during late drug development. Under the current situation, biomarkers including pharmacodynamics (PD) and safety play an important role. Hayashi et al. proved that the use of a biomarker resulted in higher success rates during anticancer drug development [1]. The discovery and use of biomarkers have been facilitated by recent advancements in omics technology, establishment of databases and understanding of pathophysiology - among others [2-4]. In the field of quantitative bioanalysis in drug development, scientists have been working on the delivery of reliable quantitative data on biomarkers with limited resources in a timely manner. However, there appears to be room for improvement.

To deliver quantitative data of high quality, scientists conduct assay validation. While some biomarker assays use the same methodologies (e.g., ligand-binding assays and chromatographic assays) as pharmacokinetics (PK) assays, there are some differences in points to consider in assay validation between biomarker assays and PK assays. Therefore, biomarker assay validation has been a hot topic in bioanalysis conferences and communities. American Association of Pharmaceutical Sciences and Clinical Ligand Assay Society organized a workshop on this subject in 2003, and the meeting outcome was published in 2006 [5]. European Bioanalysis Forum formed its biomarker team in 2010 [6]. In 2015, Japan Bioanalysis Forum Biomarker Task Force prepared a concept paper on the biomarker analysis during late clinical development [7], and American Association of Pharmaceutical Sciences and US FDA held Crystal City IV meeting [8].

However, to our knowledge, there has been only one survey assessing the status of biomarker assays in industry. The only survey was conducted within European and North American CROs by Global CRO Council for Bioanalysis (GCC) in 2012 [9]. To investigate the current situation in the pharmaceutical companies, the biomarker working group (WG) in the bioanalytical assay validation study group, which was subsidized by the Japan Agency for Medical Research and Development, decided to conduct a questionnaire survey in Japan [10]. A part of the 
survey results were already presented at the ninth Japan Bioanalysis Forum symposium in Tokyo, Japan in 2018 and twelfth Workshop on Recent Issues in Bioanalysis in PA, USA in 2018.

\section{Survey methods}

A survey in an Excel format in Japanese language was prepared by biomarker WG and distributed to all the member companies of Japan Pharmaceutical Manufacturers Association (JPMA; 71 companies) in July 2017. Generic pharmaceutical companies and CROs are not members of JPMA. All survey responses were collected by September 2017 and blinded by the secretariat of biomarker WG before analysis.

The types of survey questions were company-based questions, new medical entity (NME)-based questions and assay-based questions. For the company-based questions, only one response was collected from each company. For the other two questionnaire groups, each company was encouraged to provide multiple responses.

\section{Survey results}

\section{Company-based questions}

Total of 37 companies responded to the survey. Of these respondent companies, 26 identified themselves as Japanese companies and 11 identified as non-Japanese companies.

First, we asked them about their experiences of biomarker assays in and after 2012. Approximately a third $(32.4 \%)$ of all the respondent companies had submitted the quantitative biomarker data in new drug application (NDA), whereas only a small percentage (5.4\%) of all the respondent companies received questions on the validity of biomarker assays and sample analysis during the interaction with the regulatory agency. Most $(62.2 \%)$ of all the respondent companies had conducted biomarker (except for laboratory tests and analysis of DNA, RNA and metal) analysis for clinical trials. This result was similar between the Japanese and non-Japanese companies. Of those who conducted clinical biomarker analysis, $34.8 \%$ had one NME for the clinical biomarker analysis, whereas other companies had multiple NMEs.

Then, we asked about their standard operating procedures (SOPs) on the clinical biomarker analysis. Some $(19.2 \%)$ of the Japanese companies and most (63.6\%) of the non-Japanese companies had SOPs, indicating clear differences between the Japanese and non-Japanese companies. In addition, all SOPs of the Japanese companies did not provide guidance on assay validation (such as test items and acceptance criteria), whereas majority of the SOPs of the non-Japanese companies did so. Interestingly, two-thirds of the Japanese companies without SOPs considered that it is necessary to have SOPs somewhere in the future, whereas a third of them did not.

\section{NME-based questions}

Total of 52 NMEs were included in the present survey. It is not rare (26.9\%) that the clinical development of one NME was associated with multiple biomarkers.

\section{Assay-based questions}

In the present survey, the total number of biomarker assays was 82 . Vast majority (82.9\%) of the assays were for PD biomarkers, and only a few (3.7\%) were for safety biomarkers. The other assays (13.4\%) included those for patient stratification. For the intended use of biomarker data, $32.9 \%$ of the assays were for claiming characteristics of NME at NDA submission and the others were for exploration (29.3\%) or sponsor decision-making (35.4\%).

Ligand-binding assays and chromatographic assays accounted for 62.2 and $20.7 \%$ of the assays, respectively. The other assays (17.1\%) included flow cytometry, immunohistochemistry and enzymatic methods. Majority (74.5\%) of the ligand-binding assays used commercial kits, whereas others $(25.5 \%)$ were developed de novo. Unlike ligand-binding assays, a vast majority (82.4\%) of chromatographic assays were developed de novo.

Reference standards used in ligand-binding assays were mostly recombinant proteins (64.9\%), followed by a surrogate analyte such as the signature peptide of protein analytes $(13.5 \%)$ and the same chemical as an analyte (5.4\%). Vast majority $(88.2 \%)$ of the reference standards used in chromatographic assays were the same chemical as an analyte.

Quantitative biomarker analysis is most likely to involve the preparation of samples at known concentrations, such as calibration samples. As biomarkers are endogenous, the same biological matrix as study samples often contains a variable concentration of analyte, and is therefore, not always the best matrix used for this purpose. According to the present survey, the same biological matrix as study samples was used in $39.0 \%$ of the assays, whereas the surrogate matrix (such as water, buffer, solution included in commercial kits, protein solution, stripped 
matrix) was used in $37.8 \%$ of the assays. For the other assays (23.2\%), the respondents indicated that this question was not applicable to the assay or did not provide an answer.

The survey results on the validation items are shown in Supplementary Figure 1 (per methodology). For more than half of the ligand-binding assays, respondents examined selectivity, calibration curve, LLOQ, upper limit of quantification, accuracy, 'precision in the same matrix as study samples', matrix stability and dilution linearity/dilution integrity. For more than half of the chromatographic assays, respondents examined selectivity, calibration curve, LLOQ, upper limit of quantification, accuracy, 'precision in surrogate matrix', 'precision in the same matrix as study samples', matrix effect, carryover, matrix stability and dilution linearity/dilution integrity. Parallelism has been deemed as an important validation item in some white papers [8,9,11]. Evaluation of parallelism is considered critical if the surrogate matrix or surrogate analyte (e.g., a recombinant protein and a stable isotopelabeled analyte) is used. In the present survey, surrogate matrix and recombinant protein were used in 37.8 and $34.1 \%$ of the assays, respectively; however, parallelism was examined only in few cases ( $16.7 \%$ of the ligand-binding assays and $0.0 \%$ of the chromatographic assays). A free-text question revealed that recovery, hook effect, processed sample stability and cross validation (between different methods or different laboratories) were examined in some cases.

To investigate the determinant of validation items, we analyzed the survey result from different angles; intended use of data (i.e., exploratory purpose, sponsor decision-making and NDA submission; Supplementary Figure 2) and origin of a company (Japanese vs non-Japanese company; Supplementary Figure 3). While accuracy was examined in vast majority (81.3-93.8\%) of the assays for sponsor decision-making and NDA submission, this item was validated less frequently (60.9\%) for exploratory biomarker assays. Similarly, 'precision in surrogate matrix' was examined more frequently (50.0-56.3\%) for the assays for sponsor decision-making and NDA submission in comparison with those for exploration (30.4\%). Interestingly, specificity, matrix effect, carryover, minimum required dilution, parallelism and standard solution stability were investigated more frequently for the assays on sponsor decision-making than those for exploratory purpose and NDA submission. For the other items, executing validation appeared to be independent of the intended use of data. Validation items that non-Japanese companies conducted more frequently (at least $20 \%$ differences) than Japanese companies were specificity ( 75.0 vs $26.5 \%$ ), accuracy (100 vs $73.5 \%$ ), carryover ( 50.0 vs $24.5 \%$ ), dilution linearity/integrity ( 83.3 vs $63.3 \%)$, parallelism (33.3 vs $10.2 \%)$ and standard solution stability ( 50.0 vs $16.3 \%)$. For the other validation items, the survey results showed similar frequencies between Japanese and non-Japanese companies. We should keep in mind that the total number of answers from non-Japanese companies was only six to eight. In addition, respondents were asked if a development stage of NME affected the selection of validation items. The answer was 'no' in almost all the assays (97.7\%).

Acceptance criteria were defined in advance for the majority (76.8\%) of the assays. A free-text questionnaire revealed that some respondents consulted with the US FDA, European Medicines Agency and Ministry of Health, Labour and Welfare (of Japan) guidelines on PK assay validation for defining the acceptance criteria and they did not mention white papers on biomarker assay validation at all. A part of the respondents who set the criteria used acceptance criteria lenient than those for PK assay validation.

\section{Discussion}

The present survey in Japan revealed the current situation of biomarker assays in clinical trials. More than half $(57.7 \%)$ of the Japanese companies have conducted clinical biomarker analysis, suggesting that clinical biomarker assays have also become common in drug development in Japan. We expect this number will increase further in near future, as biomarker use has been increasing globally [12].

While most non-Japanese companies had SOPs on biomarker analysis, this was not the case for Japanese companies. The companies' size and policy, and prioritized therapeutic areas may partially explain this difference; however, we could not determine the reasons. Many Japanese companies that did not have SOPs considered that it is necessary to have SOPs somewhere in the future.

Safety biomarkers are used for assessing patients' safety in clinical trials, and therefore, draw attention from a broad range of stakeholders. In this context, a few articles state that assay validation of safety biomarkers should be more extensive than that of PD biomarkers $[6,13]$. The present survey revealed additional differences in the characteristics between PD biomarkers and safety biomarkers; PD biomarkers were analyzed much more frequently than safety biomarkers.

For an intended use of biomarker data, a third of the assays were conducted to support the regulatory review of NMEs. In other words, assays for exploration and sponsor decision-making were in majority, suggesting that it is 
prudent to discuss the level of validation of the assays for exploration and sponsor decision-making carefully. In 2006, Lee et al. proposed fit-for-purpose approach for the biomarker assay validation (such as exploratory assay validation and advanced assay validation), wherein rigor of validation depends on the intended use of biomarker data [5]. This approach assists the pharmaceutical companies conserving resources for the exploration of biomarkers and sponsor decision-making. Fit-for-purpose approach is supported by FDA guidance 2018 [14]. While the guidance requires full validation of biomarker assays to support the regulatory decision-making, it allows the industry to decide the extent of assay validation for exploratory assays.

According to the survey results, the majority of biomarker assays consisted of three types as follows: chromatographic assays to be developed de novo, ligand-binding assays to be developed de novo and ligand-binding assays using commercial kits. This finding was important, as points to consider in assay validation depend on methodology. In addition, usage of commercial kits need some consideration [15]. For example, it is a good practice to confirm that a commercial kit measures an analyte of interest by experimentation. It is recommended to focus on the above-mentioned three types of biomarker assays during initial discussion on biomarker assay validation. Henceforth, it will be necessary to discuss other methodologies (including flow cytometry and polymerase chain reaction assays) and newly developed technologies (including large molecule analysis by LC-MS).

The present survey revealed that surrogate matrix is used as the matrix for the preparation of samples at known concentration in some assays. This appears reasonable; if endogenous analyte concentration is detectable in the same biological matrix as the study samples, use of surrogate matrix is a common strategy to prepare calibration samples without interference from an endogenous analyte [5]. This strategy is one of the options for chromatographic assays (i.e., in the other option, calibration samples can be prepared by spiking stable isotope-labeled analytes into the same biological matrix as the study samples) [16]. However, use of surrogate matrix is essentially the only option for ligand-binding assays [17]. In addition, the present survey revealed that most biomarker assays are ligand-binding assays.

For validation items, GCC reported that the survey results collected from European and North American CROs in 2012 [9]. Respondents were asked whether they included calibration, precision and accuracy, selectivity/specificity, parallelism, storage stability, sensitivity, linearity of dilution, recovery when they validated ligand-binding assays and small molecule assays. When we compare the GCC survey results with the present survey results, we should be careful about the known and unknown differences in the methods of the two surveys. Validation items that were more frequently reported to be examined in the present survey in comparison with the GCC survey were LLOQ for ligand-binding assays (90.6 vs 56-60\%) and chromatographic assays (100 vs 60-75\%) and dilution linearity for ligand-binding assays (83.3 vs $44-60 \%)$. Validation items that were less frequently reported to be examined in the present survey than the GCC survey was parallelism for ligand-binding assays (16.7 vs 60-67\%) and chromatographic assays ( 0 vs $25-30 \%$ ).

Lee $e t$ al. suggested to estimate LLOQ for exploratory assay validation and establish LLOQ for advanced assay validation [5], whereas Cummings et al. and Chau et al. suggested to include LLOQ for all the assays [18,19]. Lee et al. suggested to include dilution linearity and parallelism for both assay validation [5], whereas Cummings et al. and Chau $e t$ al. suggested to include dilution linearity and parallelism only for definitive and relative quantitative assays $[18,19]$. The differences among the three articles might be linked to the differences between the present survey and the GCC survey. It is noteworthy that recent discussions in workshops and points to consider document emphasize the importance of parallelism [8,11]. We should keep in mind that it is not always possible to include parallelism in assay validation due to limited sample availability.

In the present survey, development stage did not affect the validation items. This is in line with the European Bioanalysis Forum white paper [6].

Respondents in the present survey did not mention white papers in biomarker assay validations when they were asked about documents they consult with. Those in the GCC survey mentioned regulatory guidelines on PK assay validation, as well as white papers on biomarker assay validation by Lee et al. [5], Nowatzke et al. [20], Valentin et al. [17], Cummings et al. [18] and Chau et al. [19]. Considering the differences between PK assays and biomarker assays, it is good to have points to consider or regulatory documents they can rely on about biomarker assays also in Japan.

\section{Conclusion}

The present survey in Japan revealed that biomarker assays during clinical trials have become common in drug development and approximately $30 \%$ of the assays are for regulatory decision-making. 
The majority of biomarker assays consist of three types as follows:

- Chromatographic assays to be developed de novo;

- Ligand-binding assays to be developed de novo;

- Ligand-binding assays using commercial kits.

In the future, it will be necessary to discuss other methodologies and newly developed technologies. When the respondents designate acceptance criteria, they consult PK assay guidelines and not biomarker assay white papers. The US FDA guidance 2018, issued after the present survey, provided only limited recommendations on biomarker assays. It is important to have points to consider or regulatory documents, which the Japanese bioanalysis community can embrace. While we found that parallelism was not tested very often in Japan, parallelism was conducted in most (60-67\%) of the ligand-binding assays in North American and European CROs [9]. We should discuss the necessity of parallelism in future. We hope that this survey will facilitate discussion on biomarker assay validation, and therefore promote the usage of biomarkers in drug development.

\section{Supplementary data}

See online at: https://www.future-science.com/doi/suppl/10.4155/bio-2018-0257

\section{Acknowledgements}

The authors would like to thank all survey participants for filling out the questionnaire and Japan Pharmaceutical Manufacturers Association for kind cooperation to perform this questionnaire survey.

\section{Financial \& competing interests disclosure}

This survey was supported in part by grants for Research of Regulatory Sciences on Drugs and Others, and Development of New Drugs, from the Japan Agency for Medical Research and Development. Y Ohtsu and M Katashima are employees of Astellas Pharma. T Matsumaru is an employee of Otsuka Pharmaceutical. M Kakehi is an employee of Takeda Pharmaceutical. H Kakuo is an employee of Taiho Pharmaceutical. M Mabuchi is an employee of Mitsubishi Tanabe Pharma. T Nakamura is an employee of Shin Nippon Biomedical Laboratories. S Tanaka is an employee of Aska Pharmaceutical. The authors have no other relevant affiliations or financial involvement with any organization or entity with a financial interest in, or financial conflict with, the subject matter or materials discussed in the manuscript other than those disclosed.

No writing assistance was utilized in the production of this manuscript.

\section{Disclaimer}

The opinions and perspectives presented in this paper are personal and not the official statement of National Institute of Health Sciences or Ministry of Health, Labour and Welfare of Japan.

\section{References}

Papers of special note have been highlighted as: • of interest; $\bullet \bullet$ of considerable interest

1. Hayashi K, Masuda S, Kimura H. Impact of biomarker usage on oncology drug development. J. Clin. Pharm. Ther. 38(1), 62-67 (2013).

2. Kamitsuji S, Matsuda T, Nishimura K et al. Japan PGx Data Science Consortium Database: SNPs and HLA genotype data from 2994 Japanese healthy individuals for pharmacogenomics studies. J. Hum. Genet. 60(6), 319-326 (2015).

3. Tohkin M, Ishiguro A, Kaniwa N, Saito Y, Kurose K, Hasegawa R. Prediction of severe adverse drug reactions using pharmacogenetic biomarkers. Drug Metab. Pharmacokinet. 25(2), 122-133 (2010).

4. Uehara T, Ono A, Maruyama T et al. The Japanese toxicogenomics project: application of toxicogenomics. Mol. Nutr. Food Res. 54(2), 218-227 (2010).

5. Lee JW, Devanarayan V, Barrett YC et al. Fit-for-purpose method development and validation for successful biomarker measurement. Pharm. Res. 23(2), 312-328 (2006).

-. Fundamental concept of biomarker assay validation was proposed.

6. Timmerman P, Herling C, Stoellner D et al. European Bioanalysis Forum recommendation on method establishment and bioanalysis of biomarkers in support of drug development. Bioanalysis 4(15), 1883-1894 (2012).

7. Iijima $\mathrm{K}$, Eguchi $\mathrm{C}$, Kakehi $\mathrm{M}$ et al. The JBF draft concept paper on quantitative analysis of human-endogenous substance as biomarkers in pharmaceutical development (Japanese). Presented at: 6th JBF Symposium. Tokyo, Japan, 25-26 February 2015.

8. Arnold ME, Booth B, King L, Ray C. Workshop report: Crystal City VI - bioanalytical method validation for biomarkers. AAPS J. 18(6), 1366-1372 (2016). 
9. Hougton R, Gouty D, Allinson J et al. Recommendations on biomarker bioanalytical method validation by GCC. Bioanalysis 4(20), 2439-2446 (2012).

10. Saito Y, Katori N, Ohtsu Y. Current situation on biomarker validation in Japan. Bioanalysis 10(12), 901-903 (2018).

11. Critical Path Institute. Points to Consider Document (Draft): Scientific and regulatory considerations for the analytical validation of assays used in the qualification of biomarkers in biological matrices (2017). https://healthpolicy.duke.edu/events/public-workshop-scientific-and-regulatory-considerations-analytical-validation-assays-used

- $\quad$ Fit-for-purpose acceptance criteria were proposed.

12. Hayashi K, Masuda S, Kimura H. Analyzing global trends of biomarker use in drug interventional clinical studies. Drug Discov. Ther. 6(2), 102-107 (2012).

13. Stevenson L, Garofolo F, DeSilva B et al. 2013 White paper on recent issues in bioanalysis: 'hybrid' - the best of LBA and LCMS. Bioanalysis 5(23), 2903-2918 (2013).

14. Guidance for Industry - Bioanalytical Method Validation. US Department of Health and Human Services, US FDA, Rockville, MD, USA (2018).

- This is the first regulatory document describing biomarker assay validation.

15. Khan MU, Bowsher RR, Cameron $M$ et al. Recommendations for adaptation and validation of commercial kits for biomarker quantification in drug development. Bioanalysis 7(2), 229-242 (2015).

16. Jones BR, Schultz GA, Eckstein JA, Ackermann BL. Surrogate matrix and surrogate analyte approaches for definitive quantitation of endogenous biomolecules. Bioanalysis 4(19), 2343-2356 (2012).

17. Valentin M-A, Ma S, Zhao A, Legay F, Avrameas A. Validation of immunoassay for protein biomarkers: bioanalytical study plan implementation to support pre-clinical and clinical studies. J. Pharm. Biomed. Anal. 55(5), 869-877 (2011).

18. Cummings J, Raynaud F, Jones L, Sugar R, Dive C. Fit-for-purpose biomarker method validation for application in clinical trials of anticancer drugs. Br. J. Cancer 103(9), 1313-1317 (2010).

19. Chau CH, Rixe O, McLeod H, Figg WD. Validation of analytic methods for biomarkers used in drug development. Clin. Cancer Res. 14(19), 5967-5976 (2008).

20. Nowatzke W, Cole TG, Bowsher RR. Systematic analytical validation of commercial kits for the determination of novel biomarkers for clinical drug development. Bioanalysis 2(2), 237-247 (2010). 